\title{
Exposure to Reactive Oxygen Species and Piperacillin Leads to Multidrug Resistance in Pseudomonas aeruginosa PAO1
}

\author{
Sachiko Hayakawa ${ }^{1,2^{*}}$, Emiko Furukawa ${ }^{1}$, Masato Kawamura ${ }^{1}$, Toshiaki Kikuchi ${ }^{3}$, Taizou Hirano ${ }^{4}$, Akira Watanabe ${ }^{5}$ and Shigeru Fujimura ${ }^{1,5}$ \\ ${ }^{1}$ Division of Clinical Infectious Disease \& Chemotherapy, Tohoku Medical and Pharmaceutical University, Sendai, Japan \\ ${ }^{2}$ Department of Pharmacy, Tohoku Medical and Pharmaceutical University Hospital, Sendai, Japan \\ ${ }^{3}$ Department of Respiratory Medicine and Infectious Diseases, Niigata University Graduate School of Medical and Dental Sciences, Niigata, Japan \\ ${ }^{4}$ Department of Respiratory, Tohoku University Graduate School of Medicine, Sendai, Japan \\ ${ }^{5}$ Resarch Division of Development of Anti-Infective Agents, Institute of Development Aging and Cancer, Tohoku University, Sendai, Japan
}

*Corresponding author: Sachiko Hayakawa, Division of Clinical Infectious Disease \& Chemotherapy, Tohoku Medical and Pharmaceutical University, Sendai, Japan, Tel: +81-22-259-1221; Fax: +81-22-259-1232; E-mail: s-hayakawa@hosp.tohoku-mpu.ac.jp

Received date: October 15, 2016; Accepted date: October 31, 2016; Published date: November 20, 2016

Copyright: ( $) 2016$ Hayakawa S, et al. This is an open-access article distributed under the terms of the Creative Commons Attribution License, which permits unrestricted use, distribution, and reproduction in any medium, provided the original author and source are credited.

\begin{abstract}
Pseudomonas aeruginosa is one of the most common causes of nosocomial infections. Hospital associated infection by multidrug resistant (MDR) strains constitute a serious problem worldwide. The aim of the present study was to evaluate whether exposure to sub-MIC levels of anti-pseudomonas antibiotics and reactive oxygen species (ROS), such as hydroxyl radicals, could lead to MDR of $P$. aeruginosa. $P$. aeruginosa standard strain PAO1 was used in this study. All five anti-pseudomonas agents, that is, piperacillin, levofloxacin, meropenem, ceftazidime and amikacin, were investigated for induction of resistance and cross-resistance in vitro. The reference strain was incubated $24 \mathrm{~h}$ and transferred 5 times after being exposed to $1 \mathrm{mM} \mathrm{H}_{2} \mathrm{O}_{2}$ in addition to a sub-MIC of each antibiotic by the agar dilution method. When cross-resistance to another antibiotic was confirmed, ampC, mexAB, and oprD expression and mutation of QRDR were investigated. Sub-MIC of piperacillin induced resistance to piperacillin and levofloxacin under stimulation with ROS. The mechanism of multi-resistance to $\beta$-lactams and levofloxacin was confirmed by RT-PCR. It was a decrease of oprD expression $(p<0.05)$. The increase of MIC was inhibited by the ROS scavenger sodium zinc histidine dihydrolipoyl histidinate (DHL-His-Zn).
\end{abstract}

In conclusion, for $P$. aeruginosa PAO1 to acquire multidrug resistance, stimulation with ROS was as important as exposure to sub-MIC of piperacillin.

Keywords: Multidrug resistance; ROS; Pseudomonas aeruginosa; Piperacillin; Levofloxacin; oprD; Sodium zinc histidine dihydrolipoyl histidinate

\section{Introduction}

Pseudomonas aeruginosa belongs to the bacterial family Pseudomonadaceae and is a main causative organism of healthcareassociated infection; it colonizes a wet environment such as a hospital or nursing home for a long term [1]. The compromised host easily contracts a disease such as sepsis, pneumonia, or urinary tract infection due to these bacteria. The failure of treatment because of the drug-resistant bacteria has become a serious problem recently. Sligl et al. reported the epidemiology, antimicrobial susceptibilities and outcomes of intensive care unit (ICU)-acquired gram-negative bacteremia. According to this retrospective survey of a period going from 2004 through 2012, adequate empiric therapy was related to the high mortality of patients (35\% of 30-day mortality rate), and $18 \%$ of those bacteria were $P$. aeruginosa. Additionally the susceptibility rate of each anti-pseudomonas agent was $67 \%$ for piperacillin/tazobactam, $53 \%$ for ciprofloxacin, and 53\% for imipenem [2]. The percentage of ESBL-producing Enterobacteriaceae, carbapenem-resistant Enterobacteriaceae (CRE), multidrug resistant Pseudomonas aeruginosa (MDRP), multidrug resistant Acinetobacter baumannii (MDR-AB) isolated from patients with peritoneal infections, urinary tract infection, ventilator-associated pneumonia and bacteremia has continued increasing in the United States since 2000. The increase of these drug-resistant bacteria is associated to the rise in mortality due to infectious diseases, increase of medical expenses, extension of hospitalization, and duration of stay in the ICU [3]. According to the survey of $P$. aeruginosa in 12 institutions of United States, France, Germany, Italy, and Spain, 30.5\% of patients with pneumonia were infected with MDR strains, and that was one of the factors related to the increase of hospital mortality [4]. One of the causes of the appearance of drug-resistant bacteria is selection of the resistant strain by an inappropriate antimicrobial use [5-7]. As for the mechanisms of antimicrobial resistance of $P$. aeruginosa, gene mutation of quinolone resistance-determining regions (QRDR), decrease of D2 porin, excessive expression of efflux pump, overproduction of $\beta$-lactamase by over expression of AmpC, and biofilm production, are known. Additionally, antimicrobial resistance is associated with acquisition of the metallo- $\beta$-lactamase gene, expression of the aminoglycoside modified enzyme [7-9]. Besides, it was reported that Escherichia coli, an Enterobacteriacae, suffered a mutation when exposed to reactive oxygen species (ROS) or an antimicrobial agent at a concentration below its MIC [10], and its antimicrobial susceptibility was decreased [11-13]. There have been a few reports about the effect of ROS on the acquisition of drug-resistance by $P$. aeruginosa, and most in vitro studies about antibiotic-resistance have been conducted using a bacterial strain and an antimicrobial agent $[14,15]$. However, the 
Citation: Hayakawa S, Furukawa E, Kawamura M, Kikuchi T, Hirano T, et al. (2016) Exposure to Reactive Oxygen Species and Piperacillin Leads to Multidrug Resistance in Pseudomonas aeruginosa PAO1. Clin Microbiol 5: 264. doi:10.4172/2327-5073.1000264

Page 2 of 5

bacteria are exposed to anti-pseudomonas agents and ROS derived from white blood cells for a certain period of time. In this study, we investigated the tendency and mechanism of acquisition of multidrug resistance by $P$. aeruginosa using an in vitro experimental model in which ROS are added to anti-pseudomonas agents. Furthermore, the inhibition of multidrug resistance by sodium zinc dihydrolipoyl histidinate (DHL-His-Zn), $\alpha$-lipoic acid derivative that is known as a ROS scavenger [16-19], was confirmed.

\section{Material and Methods}

\section{Bacterial strain and antibiotic susceptibilities}

The reference $P$. aeruginosa PAO1 strain was used in this study. MICs of piperacillin, levofloxacin, meropenem, ceftazidime, and amikacin were determined using E-test (AB Biodisk, Solna, Sweden). Briefly, the bacteria were suspended in $3 \mathrm{~mL}$ of saline to achieve a turbidity equivalent to that of a No. 0.5 McFarland standard and were spread on Mueller-Hinton agar (Eiken, Tokyo). After having positioned an E-test strip on the agar plate, this was cultured at $35 \mathrm{C}$ for 16-20 h. The break point MICs were interpreted according to EUCAST ver. 6.0 recommendations [20].

\section{In vitro selection of multidrug resistance}

A powder of amikacin (Meiji Seika Pharma Co., Ltd., Tokyo), ceftazidime (Glaxo SmithKline Co., Tokyo), meropenem (DainihonSumitomo Pharmaceutical Ltd., Osaka, Japan), piperacillin (TaishoToyama Pharmaceutical Co., Ltd., Tokyo), and levofloxacin (DaiichiSankyo Co., Ltd., Tokyo) was prepared. Each of these 5 antibiotics was investigated for in vitro induction of resistance and cross-resistance to the other 4 antibiotics. The reference strain PAO1 was incubated and transferred 5 times while being exposed to the sub-MIC of each antibiotic and/or $1 \mathrm{mM}$ hydrogen peroxide $\left(\mathrm{H}_{2} \mathrm{O}_{2}\right)$ as the source of ROS by the agar dilution method. The concentration of $\mathrm{H}_{2} \mathrm{O}_{2}$ was determined as maximum density not to inhibit the growth of $\mathrm{P}$. aeruginosa PAO1. The plates were incubated at $35 \mathrm{C}$ for $24 \mathrm{~h}$. Each MIC following five serial passages was determined by the E-test method. When PAO1 strain developed drug-resistance, we investigated whether the tendency to the appearance of a DR-PAO1 mutant was inhibited by the addition of $1 \mathrm{mM}$ sodium zinc histidine dihydrolipoyl histidinate (DHL-His-Zn, a ROS scavenger) [16-19].

\section{Quantification of mRNA expression by RT-PCR}

The PCR primers used in this study are listed in Table 1 [21].

\begin{tabular}{|c|c|c|c|}
\hline primer name & sequence $\left(5^{\prime}-3^{\prime}\right)$ & product size (bp) & reference \\
\hline gyrA-F & AGTCCTATCTCGACTACGCGAT & 378 & Akasaka et al. [ 23] \\
\hline gyrA-R & AGTCGACGGTTTCCTTTTCCAG & & Akasaka et al. [ 23] \\
\hline gyrB-F & TCTCCTCCGAGGTGAAGACT & 781 & This study \\
\hline gyrB-R & TACAGGCGCGACAGGCGCTT & & Bhattacharya et al. [ 24 ] \\
\hline parC-F & CGAGCAGGCCTATCTGAACTAT & 304 & Akasaka et al. [23] \\
\hline parC-R & GAAGGACTTGGGATCGTCCGGA & & Akasaka et al. [ 23] \\
\hline parE-F & CGGCGTTCGTCTCGGGCGTGGTGAAGGA & 592 & Akasaka et al. [23] \\
\hline parE-R & TCGAGGGCGTAGTAGATGTCCTTGCCGA & & Akasaka et al. [ 23] \\
\hline ampC-F & GGTGCAGAAGGACCAGGCACAGAT & 97 & Toma's et al. [ 21] \\
\hline ampC-R & CGATGCTCGGGTTGGAATAGAGGC & & Toma's et al. [ 21] \\
\hline mexAB-F & GGATCGTGACCCTGGAAG & 112 & This study \\
\hline mexAB-R & TTGAGGATGATGCCGTTCA & & This study \\
\hline oprD-F & CGGCGACATCAGCAACACC & 194 & Toma's et al. [21] \\
\hline oprD-R & GGGCCGTTGAAGTCGGAGTA & & Toma's et al. [21] \\
\hline
\end{tabular}

Table 1: PCR primers used in this study.

The levels of expression of $\operatorname{amp} C, \operatorname{mex} A B$, and oprD respectively encoding AmpC $\beta$-lactamase, efflux pump protein MexA, and outer membrane protein $\mathrm{OprD}$, were determined by real-time reverse transcription-PCR (RT-PCR) as previously described [21,22]. Briefly, the mutant DR-PAO1 that developed multidrug resistance was grown in $3 \mathrm{~mL}$ of nutrient broth at $36^{\circ} \mathrm{C}$ at $24 \mathrm{~h}$ and collected by centrifugation. Total RNA was eluted using the Aurum ${ }^{\mathrm{TM}}$ Total RNA minikit (BIO-RAD, Tokyo). Then 50 ng of purified RNA was used for one-step reverse transcription and RT-PCR amplification, using the iScript $^{\mathrm{TM}}$ One-Step RT-PCR kit with SYBR green (BIO-RAD) and a SmartCycler CFX96 (BIO-RAD). The reaction mix preparation and the thermal cycling protocol were as instructed by the manufacturer. The
mRNA transcription of $\operatorname{amp} C, \operatorname{mex} A B$, and $\operatorname{opr} D$ was determined by qRT-PCR as described previously with some modifications. Relative quantities of gene expression were calculated using the standard curve method. Expression of $16 \mathrm{~S}$ ribosomal RNA was used to normalize the transcriptional level of target genes. This experiment was performed in duplicate. Increases or decreases in normalized gene expression of $>2$ fold and $<0.5$ fold were taken as significant changes. Decreased oprD expression was considered relevant when it was $\leq 30 \%$ compared with that of $P$. aeruginosa PAO1. 
Citation: Hayakawa S, Furukawa E, Kawamura M, Kikuchi T, Hirano T, et al. (2016) Exposure to Reactive Oxygen Species and Piperacillin Leads to Multidrug Resistance in Pseudomonas aeruginosa PAO1. Clin Microbiol 5: 264. doi:10.4172/2327-5073.1000264

Page 3 of 5

\section{Mutation of the quinolone-resistance-determining region (QRDR)}

In the DR-PAO1 mutant, mutations of the genes coding for GyrA, GyrB, ParC and ParE were verified by sequencing of these genes after amplification by PCR. The PCR primers $[23,24]$ used in this study are listed in Table 1. The PCR products of these 4 genes were sequenced employing the Dye-Terminator Cycle Sequencing method. The amplification procedure for $g y r \mathrm{~A}, \operatorname{par} \mathrm{C}$ and $\operatorname{par} \mathrm{E}$ comprised denaturation at $94 \mathrm{C}$ for $3 \mathrm{~min}$ followed by 35 cycles of denaturation for $30 \mathrm{~s}$ at $94 \mathrm{C}$, annealing for $30 \mathrm{~s}$ at $55 \mathrm{C}$, and polymerization for $1 \mathrm{~min}$ at $72 \mathrm{C}$. The amplification procedure for $g y r \mathrm{~B}$ comprised denaturation at 94C for 2 min followed by 25 cycles of denaturation for $1 \mathrm{~min}$ at $94 \mathrm{C}$, annealing for $30 \mathrm{~s}$ at $60 \mathrm{C}$, and polymerization for $1 \mathrm{~min}$ at $72 \mathrm{C}$. The reactions were performed in a final volume of $25 \mu \mathrm{L}$ with $0.625 \mathrm{U}$ of Takara Ex Taq (Takara, Shiga, Japan). The PCR products were purified using the Fast Gene ${ }^{\mathrm{TM}}$ Gel/PCR Extraction Kit and sequenced. Dye Terminator Cycle Sequencing with Quick Start Kit (GenomeLab ${ }^{\mathrm{TM}}$ ) was used for the sequencing reactions, and sequences were analyzed employing the ABI 3130xl (Applied Biosystems, Foster City, CA).

\section{Statistical analysis}

The difference in expression of ampC, mexAB, and oprD between the reference PAO1 strain and DR-PAO1 mutant was analyzed by Student's t-test. A p value of $<0.05$ was regarded as statistically significant.

\section{Results}

\section{Selected multidrug resistant mutant}

MICs of each anti-pseudomonas agent against the reference PAO1 strain were piperacillin: $2 \mu \mathrm{g} / \mathrm{mL}$, ceftazidime: $0.25 \mu \mathrm{g} / \mathrm{mL}$, meropenem: $0.25 \mu \mathrm{g} / \mathrm{mL}$, levofloxacin: $0.25 \mu \mathrm{g} / \mathrm{mL}$, and amikacin: 2 $\mu \mathrm{g} / \mathrm{mL}$, respectively.

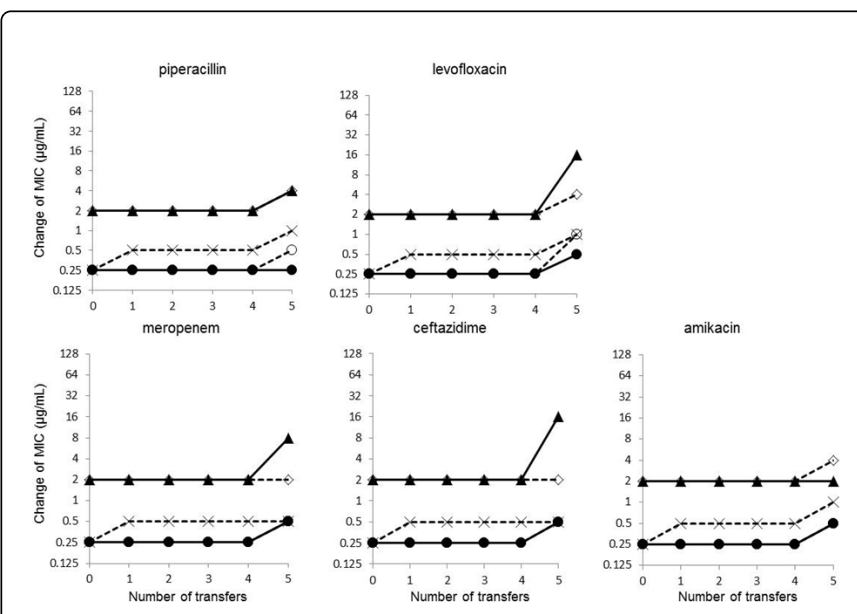

Figure 1: Change of the MIC after exposure of the reference strain to $1 / 2$ MIC of each anti-pseudomonas antimicrobial agent. Closed triangles: piperacillin, closed circles: levofloxacin, open circles: meropenem, cross: ceftazidime, open diamonds: amikacin.

Figure 1 illustrates the changes of MIC after exposure of the reference strain to the sub-MIC (1/2 MIC) of each antibiotic. After being transferred 5 times on agar plates that contained 1/2 MIC of each antibiotic, the MIC of piperacillin increased 8 folds (from 2 to 16 $\mu \mathrm{g} / \mathrm{mL}$ ) by exposure to ceftazidime and levofloxacin. It was also increased 4 folds (from 2 to $8 \mu \mathrm{g} / \mathrm{mL}$ ) by meropenem. The MIC of meropenem was increased 4 folds (from 0.25 to $1 \mu \mathrm{g} / \mathrm{mL}$ ) by levofloxacin. However, no dramatic change of susceptibility occurred after exposure to each antibiotic alone. Subsequently, a similar experiment in which hydrogen peroxide $\left(\mathrm{H}_{2} \mathrm{O}_{2}\right)$ was added as the source of ROS was performed. The PAO1 strain was exposed to the $1 / 2$ MIC of each antibiotic plus $1 \mathrm{mM} \mathrm{H}_{2} \mathrm{O}_{2}$, and the change of each MIC was assessed (Figure 2). The mutant (DR-PAO1) that acquired resistance to piperacillin and levofloxacin was selected after the fifth transfer onto agar plates containing piperacillin and hydrogen peroxide. The MICs of piperacillin and ceftazidime increased 32 folds (piperacillin: from 2 to $64 \mu \mathrm{g} / \mathrm{mL}$, ceftazidime: from 0.25 to $8 \mu \mathrm{g} / \mathrm{mL}$ ), and that of levofloxacin increased from 0.25 to $2 \mu \mathrm{g} / \mathrm{mL}$ (Figure 2). After exposure to $1 / 2 \mathrm{MIC}$ of levofloxacin and $1 \mathrm{mM} \mathrm{H}_{2} \mathrm{O}_{2}$, the MIC of levofloxacin hardly increased. In the contrast, no increase of the MIC of either antibiotic was observed after exposure to hydrogen peroxide alone (Supplemental data 1).

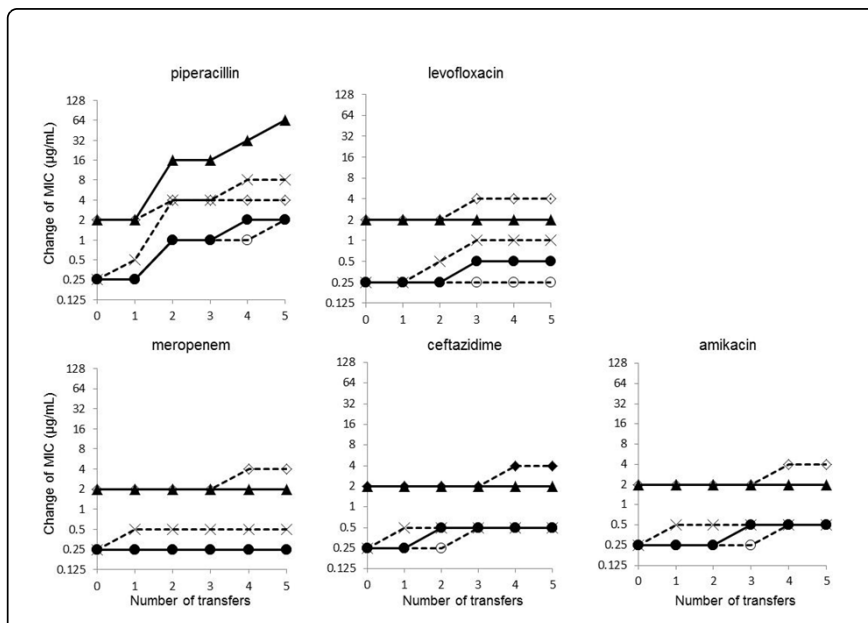

Figure 2: Change of the MIC after exposure of the reference strain to $1 / 2 \mathrm{MIC}$ of each anti-pseudomonas antimicrobial agent plus 1 $\mathrm{mM} \mathrm{H}_{2} \mathrm{O}_{2}$. Closed triangles: piperacillin, closed circles: levofloxacin, open circles: meropenem, cross: ceftazidime, open diamonds: amikacin.

\section{Inhibition of multidrug resistance by a ROS scavenger}

The increase of MIC of piperacillin and levofloxacin after exposure of the reference strain to piperacillin plus hydrogen peroxide shown in this study was completely inhibited by the addition of $1 \mathrm{mM}$ DHL-His$\mathrm{Zn}$ (Figure 3). Thus, acquisition of multidrug resistance by $P$. aeruginosa PAO1 was inhibited by DHL-His-Zn.

\section{Decrease of oprD mRNA expression}

The mRNA transcription levels of ampC, mexAB, and opr $\mathrm{D}$ of the DR-PAO1 mutant were compared with those of the standard PAO1 strain (Figure 4). The value of normalized expression of opr $\mathrm{D}$ in PAO1 strain was $1.502 \pm 0.005$, and the value in DR-PAO1 mutant was 0.996 \pm 0.004 . The mean expression level of oprD in the DR-PAO1 mutant 
decreased by $33.7 \%(\mathrm{p}<0.05)$. There were not statistical differences in mRNA expression between ampC and mexAB.
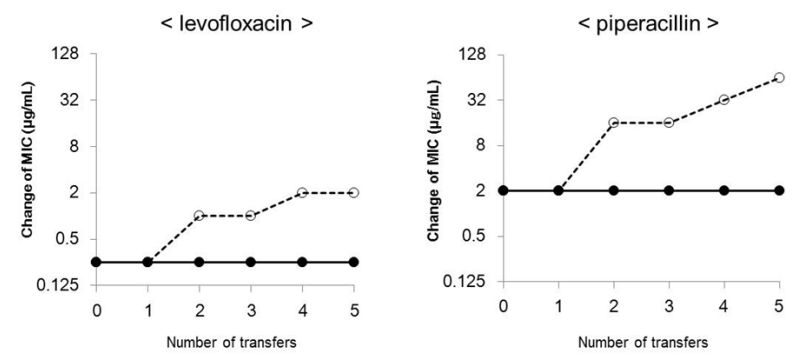

Figure 3: MIC change after exposure of the reference strain to piperacillin plus ROS, and inhibitory effect of DHL-Zn-His. The MIC of each antibiotic was increased by treatment with $1 \mu \mathrm{g} / \mathrm{mL}$ piperacillin and $1 \mathrm{mM} \mathrm{H}_{2} \mathrm{O}_{2}$. DHL-Zn-His inhibited the increase of MIC. Open circles: $1 \mu \mathrm{g} / \mathrm{mL}$ piperacillin $+1 \mathrm{mM} \mathrm{H}_{2} \mathrm{O}_{2}$, closed circles: $1 \mu \mathrm{g} / \mathrm{mL}$ piperacillin+1 $\mathrm{mM} \mathrm{H}_{2} \mathrm{O}_{2}+1 \mathrm{mM}$ DHL-His-Zn.

\section{parE Mutations}

Multiple mutations in parE gene encoding ParE of type II topoisomerase were confirmed in the DR-PAO1 mutant. The amino acid sequences of ParE showed multiple replacements of Ser-373 to Ile, Ala-375 to Asp, and Arg-378 to His. There were no amino acid changes for GyrA, GyrB and ParC in the DR-PAO1 mutant.

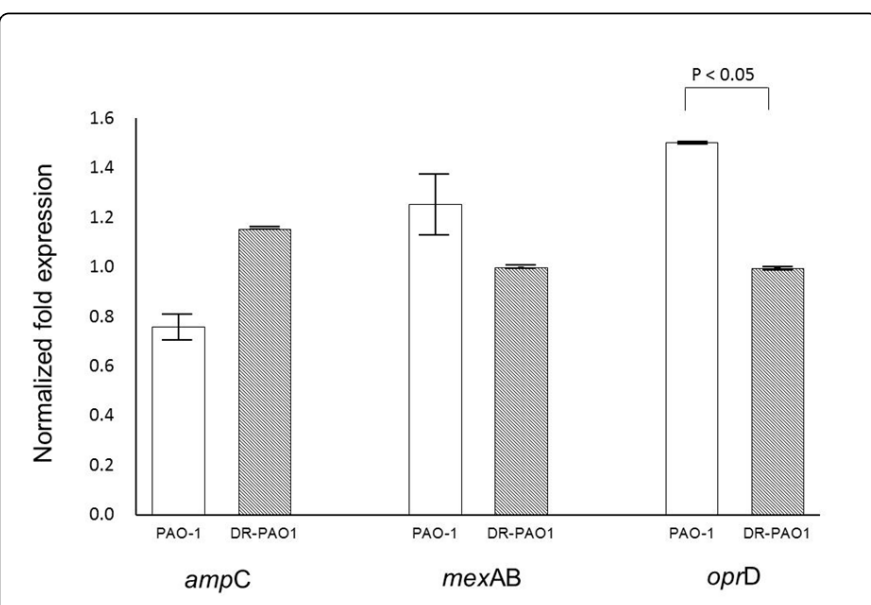

Figure 4: Comparison of mRNA expression of genes conferring $\beta$ lactam and levofloxacin resistance. White column: PAO-1 strain, gray column: drug-resistant (DR) PAO-1 strain induced by $1 \mu \mathrm{g} / \mathrm{mL}$ piperacillin $+1 \mathrm{mM} \mathrm{H}_{2} \mathrm{O}_{2}$

\section{Discussion}

ROS are generated in vivo upon energy production, immune responses against foreign substances, processing of unwanted cells, cell signaling, simultaneously oxidized lipids, proteins, and enzymes, and occasionally cause DNA damage $[10,11,25]$. Bacteria are exposed to ROS in vivo during infection. The effects of ROS on cells vary with their concentration. Specifically, they have bactericidal effects and enhance the effects of antibiotics at high concentrations [25,26], while at low concentrations they may cause gene mutations resulting in drug resistance [11-15]. In $P$. aeruginosa, hydrogen peroxide and amikacin or tobramycin cause the expression of the drug efflux pump mexXY in vitro, which reduces the susceptibility of the bacteria to aminoglycosides [27]. Clinically, $54.4 \%$ of $P$. aeruginosa isolated from sputum samples collected from patients with cystic fibrosis (CF) were mutated strains, which were more resistant to anti-pseudomonas agents (tazobactam/piperacillin and ceftazidime) than non-mutated ones. In addition, sputum samples collected from CF patients were examined over time, demonstrating that the mutated strains could be detected for more than five years after infection, and with an increased number of mutations. That is, prolonged exposure to antipseudomonas agents and ROS facilitated selection of resistant $P$. aeruginosa strains during persistent infection [28].

In the present study, cross-resistance among various antibacterial agents was examined using $P$. aeruginosa strain $\mathrm{PAO} 1$ after the addition of $\mathrm{H}_{2} \mathrm{O}_{2}$ (in vivo oxidative stress). As a result, $1 \mathrm{mM} \mathrm{H} \mathrm{H}_{2} \mathrm{O}_{2}$ alone did not alter the MIC values of the antibacterial agents against PAO-1 strain. However, the addition of piperacillin to $\mathrm{H}_{2} \mathrm{O}_{2}$ increased the MIC of levofloxacin from 0.25 to $2 \mu \mathrm{g} / \mathrm{mL}$, as well as of piperacillin, showing multidrug resistance. In this multidrug resistant strain DR$\mathrm{PAO} 1$, the expression of the opr $\mathrm{D}$ gene encoding $\mathrm{D} 2$ porin significantly decreased $(\mathrm{p}<0.05)$. In addition, amino acid mutations (S373I, A375D, and R378H), unreported in the quinolone resistance determining region (QRDR) of parE, were detected. Such mutations were not found for other $\beta$-lactams, such as cephalosporins and carbapenems. Oxidative stress induced by ROS, besides exposure to penicillin antibiotics, might cause DNA damage and induce MDR phenomena in $P$. aeruginosa.

Kohanski et al. exposed various bacterial strains to antibacterial agents, and demonstrated the production of hydroxyl radical in E. coli after the addition of penicillin-derived antibiotics [10]. Interestingly, the addition of amoxicillin, induced hydroxyl radical production in two stages (within 1 and 2-3 hours), suggesting prolonged exposure to ROS, compared with other antibiotics. Besides $1 \mathrm{mM} \mathrm{H}_{2} \mathrm{O}_{2}$, ROS produced in bacterial cells by penicillin-derived antibiotics might suppress the oprD expression and cause the parE mutation, resulting in cross-resistance to quinolones.

The results of the present study suggest that at a low dose the antipseudomonas agent piperacillin may induce cross-resistance to levofloxacin when combined with ROS in vivo. In addition, it should be noted that the MIC of meropenem was increased on the same condition, although the increase did not result in resistance to this drug.

The in vitro MDR phenomenon, observed in the present study, was suppressed by the addition of the ROS inhibitor DHL-His-Zn ( $\alpha$-lipoic acid derivative). This agent is an antioxidant in which histidine and zinc are bound to $\alpha$-lipoic acid. Its clinical applications, such as suppression of hair loss due to oxidative stress induced by antineoplastic agents in rats and suppression of in vitro colon cancer cell growth, have been investigated [17,19]. Traditionally, in vitro studies on bacterial antibiotic resistance have been conducted using bacteria and antibiotics. The present study also included ROS as a biological factor, providing an in vitro experimental system reflecting in vivo conditions. In the present situation, new multidrug resistant bacteria are increasing in the world, drug resistance suppression by combining DHL-His-Zn may improve the therapeutic outcomes of intractable infections. Anti-ROS agents should be investigated to solve 
the global problem caused by multidrug resistant Gram-negative bacilli.

In conclusion, we demonstrated multidrug resistance acquisition in $P$. aeruginosa stimulated with a low dose of piperacillin and oxidative stress using ROS as a biological factor. Although high-dose penicillin treatments have recently been recommended according to the PK-PD theory, they can be employed in a small number of countries in the world. In infectious diseases, such as deep abscesses and $\mathrm{CF}$, antibacterial agents are less likely to migrate to the sites of infection, which may result in low exposure of pathogenic bacteria to antibiotics. Under such conditions, oxidative stress causes gene mutations, which may result in the emergence of multidrug resistant bacteria. Our findings suggest, for potential infection by $P$. aeruginosa, high doses of antipseudomonas agents should be administered in combination with antioxidants (e.g., DHL-His-Zn) to improve therapeutic effects and prevent the emergence of multidrug resistant bacteria.

\section{Acknowledgement}

We thank Yasuko Kariya and Tomoko Ito of IDAC, Tohoku University for technical assistance.

\section{Conflict of Interest}

The authors declare that they have no competing interest.

\section{References}

1. Garvey MI, Bradley CW, Tracey J, Oppenheim B (2016) Continued transmission of Pseudomonas aeruginosa from a wash hand basin tap in a critical care unit. J Hosp Infect 94: 8-12.

2. Sligl WI, Dragan T, Smith SW (2015) Nosocomial gram-negative bacteremia in intensive care: epidemiology, antimicrobial susceptibilities, and outcomes. Int J Infect Dis 37: 129-134.

3. Kaye KS, Pogue JM (2015) Infections caused by resistant gram-negative bacteria: epidemiology and management. Pharmacother 35: 949-962.

4. Micek ST, Wunderink RG, Kollef MH, Chen C, Rello J, et al. (2015) An international multicenter retrospective study of Pseudomonas aeruginosa nosocomial pneumonia: impact of multidrug resistance. Crit Care 19: 219.

5. Tsai MH, Wu TL, Su LH, Lo WL, Chen CL, et al. (2014) Carbapenemresistant-only Pseudomonas aeruginosa infection in patients formerly infected by carbapenem-susceptible strains. Int J Antimiclob Agents 44: 541-545.

6. Alcalá-Franco B, Montanari S, Cigana C, Bertoni G, Oliver A, et al. (2012) Antibiotic pressure compensates the biological cost associated with Pseudomonas aeruginosa hypermutable phenotypes in vitro and in a murine model of chronic airways infection. J Antimicrob Chemother 67: 962-969.

7. Santajit S, Indrawattana N (2016) Mechanisms of antimicrobial resistance in ESKAPE pathogens. Biomed Res Int.

8. Poole K (2011) Pseudomonas aeruginosa: resistance to the max. Front Microbol 2: 1-13.

9. Morita Y, Tomida J, Kawamura Y (2014) Responses of Pseudomonas aeruginosa to antimicrobials. Front Microbiol 4: 1-8.

10. Kohanski MA, Dwyer DJ, Hayete B, Lawrence CA, Collins JJ (2007) A common mechanism of cellular death induced by bactericidal antibiotics. Cell 130: 797-810.

11. Dwyer DJ, Kohanski MA, Collins JJ (2009) Role of reactive oxygen species in antibiotic action and resistance. Curr Opin Microbiol 12: 482-489.
12. Kohanski MA, DePristo MA, Collins JJ (2010) Sublethal antibiotic treatment leads to multidrug resistance via radicalinduced mutagenesis. Mol Cell 37: 311-320.

13. Händel N, Hoeksema M, Freijo Mata M, Brul S, ter Kuile BH (2015) Effects of stress, reactive oxygen species, and the SOS response on de novo acquisition of antibiotic resistance in Escherichia coli. Antimicrob Agents Chemother 60: 1319-1327.

14. Jorgensen KM, Wassermann T, Jensen PØ, Hengzuang W, Molin S, el al. (2013) Sublethal ciprofloxacin treatment leads to rapid development of high-level ciprofloxacin resistance during long-term experimental evolution of Pseudomonas aeruginosa. Antimicrob Agents Chemother 57: 4215-4221.

15. Mandsberg LF, Ciofu O, Kirkby N, Christiansen LE, Poulsen HE, et al (2009) Antibiotic resistance in Pseudomonas aeruginosa strains with increased mutation frequency due to inactivation of the DNA oxidative repair system. Antimicrob Agents Chemother 53: 2483-2491.

16. Hagiwara S, Teshima Y, Takahashi N, Koga H, Saikawa T, et al. (2011) New lipoic acid derivative drug sodium zinc dihydrolipoylhistidinate prevents cardiac dysfunction in an isolated perfused rat heart model. Crit Care Med 39: 506-511.

17. Hagiwara S, Uchida T, Koga H, Inomata M, Yoshizumi F, et al. (2011) The a-lipoic acid derivative sodium zinc dihydrolipoylhistidinate reduces chemotherapy-induced alopecia in a rat model: a pilot study. Surg Today 41: 693-697.

18. Koga H, Hagiwara S, Kusaka J, Goto K, Uchino T, et al. (2012) New alipoic acid derivative, DHL-HisZn, ameliorates renal ischemiareperfusion injury in rats. J Surg Res 174: 352-358.

19. Kono Y, Inomata M, Hagiwara S, Hiratsuka T, Suzuki K, et al. (2012) Antiproliferative effects of a new a-lipoic acid derivative, DHL-HisZnNa, in HT29 human colon cancer cells in vitro. Expert Opin Ther Targets 16: S103-109.

20. The european committee on antimicrobial susceptibility testing, Breakpoint tables for interpretation of MICs and zone diameters, version 6.0 .

21. Toma's M, Doumith M, Warner M, Turton JF, Beceiro A, et al. (2010) Efflux pumps, OprD porin, AmpC -lactamase, and multiresistance in Pseudomonas aeruginosa isolates from cystic fibrosis patients. Antimicrob Agents Chemother 54: 2219-2224.

22. Juan C, Moya' B, Pérez JL, Oliver A (2006) Stepwise upregulation of the Pseudomonas aeruginosa chromosomal cephalosporinase conferring high-level $\beta$-lactam resistance involves three AmpD homologues. Antimicrob Agents Chemother 50: 1780-1787.

23. Akasaka T, Tanaka M, Yamaguchi A, Sato K (2001) Type II topoisomerase mutations in fluoroquinolone-resistant clinical strains of Pseudomonas aeruginosa isolated in 1998 and 1999: role of target enzyme in mechanism of fluoroquinolone resistance. Antimicrob Agents Chemother 45: 2263-2268.

24. Bhattacharya D, Dey S, Kadam S, Kalal S, Jali S (2015) Isolation of NDM-1-producing multidrug-resistant Pseudomonas putida from a paediatric case of acute gastroenteritis, India. New Microbe and New Infect 5: 5-9.

25. Winterbourn CC, Kettle AJ (2013) Redox reactions and microbial killing in the neutrophil phagosome. Antioxd Redox Signal 18: 642-660.

26. Ferrándiz MJ, Martín-Galiano AJ, Arnanz C, Zimmerman T, de la Campa AG (2015) Reactive oxygen species contribute to the bactericidal effects of the fluoroquinolone moxifloxacin in Streptococcus pneumoniae. Antimicrob Agents Chemother 60: 409-417.

27. Fraud S, Poole K (2011) Oxidative stress induction of the MexXY multidrug efflux genes and promotion of aminoglycoside resistance development in Pseudomonas aeruginosa. Antimicrob Agents Chemother 55: 1068-1074.

28. Ciofu O, Riis B, Pressler T, Poulsen HE, Høiby N (2005) Occurrence of hypermutable Pseudomonas aeruginosa in cystic fibrosis patients is associated with the oxidative stress caused by chronic lung inflammation. Antimicrob Agents Chemother 49: 2276-2282. 